\title{
Video Article \\ Hemogenic Reprogramming of Human Fibroblasts by Enforced Expression of Transcription Factors
}

\author{
Rita Silvério-Alves ${ }^{1,2,3}$, Andreia M. Gomes ${ }^{3}$, Ilia Kurochkin ${ }^{4}$, Kateri A. Moore ${ }^{5,6}$, Carlos-Filipe Pereira ${ }^{1,2,3}$ \\ ${ }^{1}$ Molecular Medicine and Gene Therapy, Lund Stem Cell Center, Lund University \\ ${ }^{2}$ Wallenberg Center for Molecular, Lund University \\ ${ }^{3}$ Center for Neuroscience and Cell Biology, University of Coimbra \\ ${ }^{4}$ Skolkovo Institute of Science and Technology \\ ${ }^{5}$ Department of Cell, Developmental and Regenerative Biology, Icahn School of Medicine at Mount Sinai \\ ${ }^{6}$ Black Family Stem Cell Institute, Icahn School of Medicine at Mount Sinai
}

Correspondence to: Carlos-Filipe Pereira at filipe.pereira@med.lu.se

URL: https://www.jove.com/video/60112

DOI: doi:10.3791/60112

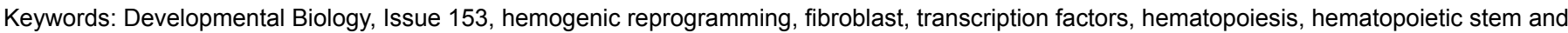
progenitor cells, endothelial-to-hematopoietic transition

Date Published: 11/4/2019

Citation: Silvério-Alves, R., Gomes, A.M., Kurochkin, I., Moore, K.A., Pereira, C.F. Hemogenic Reprogramming of Human Fibroblasts by Enforced Expression of Transcription Factors. J. Vis. Exp. (153), e60112, doi:10.3791/60112 (2019).

\section{Abstract}

The cellular and molecular mechanisms underlying specification of human hematopoietic stem cells (HSCs) remain elusive. Strategies to recapitulate human $\mathrm{HSC}$ emergence in vitro are required to overcome limitations in studying this complex developmental process. Here, we describe a protocol to generate hematopoietic stem and progenitor-like cells from human dermal fibroblasts employing a direct cell reprogramming approach. These cells transit through a hemogenic intermediate cell-type, resembling the endothelial-to-hematopoietic transition (EHT) characteristic of HSC specification. Fibroblasts were reprogrammed to hemogenic cells via transduction with GATA2, GFI1B and FOS transcription factors. This combination of three factors induced morphological changes, expression of hemogenic and hematopoietic markers and dynamic EHT transcriptional programs. Reprogrammed cells generate hematopoietic progeny and repopulate immunodeficient mice for three months. This protocol can be adapted towards the mechanistic dissection of the human EHT process as exemplified here by defining GATA2 targets during the early phases of reprogramming. Thus, human hemogenic reprogramming provides a simple and tractable approach to identify novel markers and regulators of human HSC emergence. In the future, faithful induction of hemogenic fate in fibroblasts may lead to the generation of patient-specific HSCs for transplantation.

\section{Video Link}

The video component of this article can be found at https://www.jove.com/video/60112/

\section{Introduction}

Definitive hematopoietic stem and progenitor cells (HSPCs) emerge in the aorta-gonad-mesonephros (AGM) region and placenta from endothelial precursors with hemogenic capacity, through an endothelial-to-hematopoietic transition (EHT) ${ }^{1,2}$. Hemogenic precursors (HPs) express both endothelial and hematopoietic markers, but their precise identification remains elusive, particularly in the human system. Despite being a relatively conserved process in mammals, hematopoietic stem cell (HSC) development still differs significantly between humans and mouse models ${ }^{3,4}$. Therefore, in vitro approaches to recapitulate human HSC development are needed.

Differentiation of pluripotent stem cells (PSCs) to HSCs, although promising, has met limited success over the past 20 years, mostly due to the available differentiation protocols, which result in primitive hematopoietic progenitors with poor engraftment ability ${ }^{5,6,7}$. Alternatively, direct cell reprogramming methodologies have been applied to generate HSPC-like cells from multiple cell types, using transcription factors $(\mathrm{TFs})^{8,9}$. In particular, the overexpression of three TFs, Gata2, Gfi1b and cFos, converted mouse embryonic fibroblasts into HSPCs through an HP intermediate with a defined phenotype (Prom1+Sca-1+CD34+CD45- $)^{10}$. This process resembled the EHT that occurs in the embryo and placenta, during specification of definitive hematopoiesis. This phenotype enabled the identification and isolation of a population of HPs in the mouse placenta that after short-term culture and Notch activation generated serially transplantable HSCs ${ }^{11}$.

So far, no phenotype has been established that distinguishes human HSCs from their precursors, but some molecules are known to be expressed in emerging HSCs. Integrin alpha 6 (ITGA6 or CD49f) is highly expressed in long-term repopulating HSCs, the most immature cells in the HSC compartment ${ }^{12}$, and angiotensin-converting enzyme (ACE or CD143) is present in CD34 negative hematopoietic precursors in embryonic blood-forming tissues ${ }^{13}$.

Recently, we have demonstrated that human versions of the three TFs, GATA2, FOS and GFI1B reprogram human dermal fibroblasts (HDFs) into HPS with short-term engraftment capacity ${ }^{14}$. In the initial phases of reprogramming, GATA2 engages open chromatin and recruits GFI1B 
and FOS to repress fibroblast genes and activate endothelial and hematopoietic genes. Induced cells highly expressed CD49f and ACE, and contained a small percentage of cells expressing the HSPC marker CD34. The CD9 gene, which is expressed in HSCs ${ }^{15}$ and is important for HSC homing ${ }^{16}$, was shown to be a direct target of GATA2 and among the most up-regulated genes in reprogrammed cells ${ }^{14}$. CD9 may therefore constitute an additional marker for HPs of human definitive hematopoiesis.

In this protocol, we describe the generation of HSPC-like cells from human fibroblasts through enforced expression of GATA2, GFI1B and FOS, as well as an adapted method for chromatin immunoprecipitation (ChIP)-sequencing (seq) analysis at the onset of reprogramming. TFs were encoded in a doxycycline (DOX)-inducible lentiviral vector ( $p F U W$-tetO) that contains a tetracycline response element (TRE) and a minimal CMV promoter, and were transduced together with a constitutive vector containing the reverse tetracycline transactivator protein ( $\mathrm{pFUW-M2rtTA)}$. When DOX (analog of tetracycline) is added after transduction, it binds to the rtTA protein which interacts with the TRE allowing TF transcription (Tet-On system). The procedure requires 25 days to complete. For ChIP-seq experiments, HDFs were transduced with tagged versions of GATA2 (pFUW-tetO-3xFLAG-GATA2) and GFI1B (pLV-tetO-HA-GFI1B), plus pFUW-tetO-FOS and TF binding sites were analyzed two days after DOX supplementation.

Ultimately, hemogenic reprogramming of human fibroblasts provides an in vitro tractable system to study the mechanisms underlying human developmental hematopoiesis and a potential source of patient-specific HSPCs for future clinical application.

\section{Protocol}

This protocol was performed according to Lund University's human research ethics committee guidelines and should be done in accordance with individual institutional guidelines.

\section{Reagent Preparation}

1. For Dulbecco's modified Eagle's medium (DMEM)/20\% fetal bovine serum (FBS), mix high glucose DMEM containing sodium pyruvate with $20 \%$ FBS, $1 \%$ penicillin-streptomycin (pen/strep), $1 \%$ L-glutamine, $1 \%$ non-essential amino acids and $10^{-4}$-M 2-mercaptoethanol.

2. For complete DMEM, mix high glucose DMEM containing sodium pyruvate with $10 \% \mathrm{FBS}, 1 \%$ Pen/strep and $1 \% \mathrm{~L}$-glutamine.

3. For hematopoietic medium, mix hematopoietic medium (Table of Materials) with $10^{-6} \mathrm{M}$ hydrocortisone and $1 \%$ pen/strep.

4. Use phosphate-buffered saline (PBS) without calcium or magnesium.

\section{Human Dermal Fibroblast Isolation}

NOTE: HDFs can be purchased from certified suppliers (Table of Materials). In that case, expand fibroblasts and use them directly in reprogramming experiments (section 4). Alternatively, HDFs can be isolated from donors. If fibroblasts are isolated from different donors, keep the samples separated from each other at all steps of the protocol. Label plates/wells and collection tubes with the identification number of each donor.

1. Obtain HDFs from $3 \mathrm{~mm}$ round skin punch biopsies performed by qualified physicians.

2. Coat three wells of a tissue culture-treated 6-well plate with $500 \mu \mathrm{L}$ of $0.1 \%$ gelatin and incubate for $20 \mathrm{~min}$ at $37^{\circ} \mathrm{C}$.

3. Aspirate the remaining gelatin solution and add $750 \mu \mathrm{L}$ of DMEM/20\% FBS to each well. The entire surface of the well should be covered with medium.

4. Add $1.5 \mathrm{~mL}$ of DMEM/20\% FBS to the inside surface of a sterile $100 \mathrm{~mm}$ petri dish lid and spread the drop with the aid of a $5 \mathrm{~mL}$ serological pipette.

5. Place the skin biopsy in the medium on the lid with sterilized forceps.

6. Dissect the skin biopsy into nine identical sections, using one sterilized scalpel to hold the biopsy in place and a second scalpel to cut.

7. Place three biopsy pieces per well using pointed forceps. Make sure the pieces attach to the bottom of the well.

8. Lay a $22 \mathrm{~mm}$ coverslip on top of the pieces and apply some pressure.

9. Incubate the plate at $37^{\circ} \mathrm{C}, 5 \% \mathrm{CO}_{2}$, for a week. Check cells daily and add $200 \mu \mathrm{L}$ of DMEM/20\% FBS every 2 days to replace evaporated medium.

10. After one week, add up to $2 \mathrm{~mL}$ of DMEM/20\% FBS and replace medium every 2-3 days.

11. Passage cells at 1:4 ratio when wells are confluent (about 4-8 weeks).

1. Prepare $0.1 \%$ gelatin coated tissue culture-treated 6 -well plates.

2. Aspirate medium from wells at $80 \%$ confluency and wash once with $1 \mathrm{~mL}$ of PBS.

3. Remove coverslip with sterile forceps and place the coverslip into a new well of a 6-well plate, with the tissue side up. NOTE: Cells that remained attached to the coverslip will also be harvested.

4. Add $500 \mu \mathrm{L}$ of dissociation solution (Table of Materials) per well (including wells with the coverslips) and incubate at $37{ }^{\circ} \mathrm{C}, 5 \% \mathrm{CO}_{2}$ for 5-10 min. Check when cells start to rise from the bottom of the well and inactivate the dissociation solution by adding $500 \mu \mathrm{L}$ of DMEM/20\% FBS to each well.

5. Collect fibroblasts from all the wells into a $15 \mathrm{~mL}$ conical tube. Add extra medium to the wells to collect the remaining cells. Centrifuge the tube at $350 \times g$ for $5 \mathrm{~min}$

6. In the meantime, add $500 \mu \mathrm{L}$ of DMEM/20\% FBS to each well of previously gelatin coated plates.

7. Aspirate medium and resuspend fibroblasts in $6 \mathrm{~mL}$ of DMEM/20\% FBS.

8. Add $500 \mu \mathrm{L}$ of fibroblast suspension to each well (two 6 -well plates per sample/donor in total). Incubate cells overnight at $37{ }^{\circ} \mathrm{C}, 5 \%$ $\mathrm{CO}_{2}$.

12. On the next day, add $1 \mathrm{~mL}$ of DMEM/20\% FBS to each well. Replace medium with $2 \mathrm{~mL}$ of DMEM/20\% FBS every $2-3$ days until wells are $80 \%$ confluent.

13. Repeat section 2.11 for three confluent wells until third passage is reached. 
14. Freeze fibroblasts from confluent wells (passages 1 and 3 ).

1. Aspirate medium from the wells and wash once with $1 \mathrm{~mL}$ of PBS.

2. Dissociate and collect fibroblasts as described in steps 2.11 .4 and 2.11.5.

3. Count cells with a hemocytometer and centrifuge the tube at $350 \times g$ for $5 \mathrm{~min}$.

4. After centrifugation, aspirate medium and resuspend fibroblasts in FBS with $10 \%$ DMSO at a density of $5 \times 10^{5} \mathrm{cells} / \mathrm{mL}$.

5. Add $1 \mathrm{~mL}$ of the cell suspension per cryovial and freeze cells overnight at $-80^{\circ} \mathrm{C}$ using a freezing container. Move vials to $-150{ }^{\circ} \mathrm{C}$ (liquid nitrogen) for long-term storage.

\section{Lentiviral Production}

1. Grow HEK293T cells in a $100 \mathrm{~mm}$ tissue culture-treated dish with $10 \mathrm{~mL}$ of complete DMEM, at $37{ }^{\circ} \mathrm{C}, 5 \% \mathrm{CO}_{2}$, until confluency is reached.

2. On the day prior to transfection, aspirate medium and wash the dish carefully with $5 \mathrm{~mL}$ of PBS.

3. After removing PBS, add $1.5 \mathrm{~mL}$ of dissociation solution and incubate at $37^{\circ} \mathrm{C}, 5 \% \mathrm{CO}_{2}$ for $5-10$ min to dissociate cells from the dish. NOTE: It is recommended to warm both PBS and dissociation solution before using, so that cells do not suffer a thermal shock.

4. Inactivate dissociation solution with $3 \mathrm{~mL}$ of complete DMEM and transfer the cell suspension to a $15 \mathrm{~mL}$ conical tube. Wash the dish with 5 $\mathrm{mL}$ of complete DMEM to remove remaining attached cells and transfer this volume to the $15 \mathrm{~mL}$ conical tube.

5. Centrifuge cell suspension at $350 \times g$ for 5 min.

6. Aspirate supernatant and split the cell pellet evenly between six $100 \mathrm{~mm}$ tissue culture-treated dishes in a final volume of $10 \mathrm{~mL}$ of complete DMEM per dish. Cells should be approximately $60 \%$ confluent by the time of transfection.

7. On the next day, transfect cells with the plasmid mixes as follows:

NOTE: This part of the protocol describes the production of lentiviruses in one $100 \mathrm{~mm}$ tissue culture-treated dish per plasmid mix. To obtain higher volumes of lentiviral supernatant for concentration, use at least four $100 \mathrm{~mm}$ HEK293T cell culture dishes per mix.

1. In a $15 \mathrm{~mL}$ conical tube, add $10 \mu \mathrm{g}$ of the three transfer plasmids together: $3.33 \mu \mathrm{g}$ of pFUW-tetO-GATA2 (Addgene plasmid $\# 125028)^{14}, 3.33 \mu \mathrm{g}$ of pFUW-tetO-GFI1B (Addgene \#125597) ${ }^{14}$ and $3.33 \mu \mathrm{g}$ of pFUW-tetO-FOS (Addgene \#125598) ${ }^{14}$, plus $10 \mu \mathrm{g}$ of the $2^{\text {nd }}$ generation psPAX2 packaging vector encoding the Gag, Pol, Tat and Rev genes (Addgene \#12260) and $5 \mu \mathrm{g}$ of pMD2.G envelope vector encoding the VSV-G gene (Addgene \#12259). Add water up to $500 \mu \mathrm{L}$

2. In two new $15 \mathrm{~mL}$ conical tubes add $10 \mu \mathrm{g}$ of FUW-M2rtTA plasmid (Addgene \#20342) ${ }^{17}, 10 \mu \mathrm{g}$ of psPAX2 packaging vector and $5 \mu \mathrm{g}$ of pMD2.G envelope vector to each tube. Add water up to $500 \mu \mathrm{L}$. One tube is going to be used as a control.

3. To each tube add $62.5 \mu \mathrm{L}$ of $2 \mathrm{M} \mathrm{CaCl}_{2}$. Next, release bubbles into each mixture using a Pasteur pipet inserted into a pipet controller. While bubbles are forming, pipette $500 \mu \mathrm{L}$ of $\mathrm{N}, \mathrm{N}$-bis(2-hydroxyethyl)-2-aminoethanesulfonic acid (BES) buffered saline (pH 7.1, 25 $\left.{ }^{\circ} \mathrm{C}\right)$, with a $\mathrm{P} 1000$ pipette, drop-wise against the Pasteur pipet and onto the mixture.

4. Incubate tubes at room temperature for at least $15 \mathrm{~min}$. The mixtures will appear slightly cloudy after some time.

8. Meanwhile, aspirate medium from HEK293T cell dishes (passaged the day before) and add $10 \mathrm{~mL}$ of complete DMEM without antibiotics. Be careful and add medium slowly as HEK293T cells are semi-adherent.

9. Distribute each individual mixture (approximately $1 \mathrm{~mL}$ ) evenly and drop-wise into separate dishes and incubate overnight at $37{ }^{\circ} \mathrm{C}, 5 \% \mathrm{CO}_{2}$.

10. Replace medium with $4 \mathrm{~mL}$ of complete DMEM, $24 \mathrm{~h}$ after incubation. Incubate overnight at $37{ }^{\circ} \mathrm{C}, 5 \% \mathrm{CO}_{2}$. If available, incubate instead at $32{ }^{\circ} \mathrm{C}, 5 \% \mathrm{CO}_{2}$, as the reduced temperature will increase the half-life of the lentiviral particles.

11. Collect supernatant with lentiviral particles three times to a $50 \mathrm{~mL}$ conical tube. Do not mix different lentiviral particles at this point. Each dish will result in $12 \mathrm{~mL}$ of lentiviral supernatant. Four dishes of the same viral preparation fit into one $50 \mathrm{~mL}$ conical tube. CAUTION: Perform lentiviral collection in a biosafety level-2 laboratory in a laminar flow hood dedicated for lentiviral work and place viral contaminated waste (tubes, tips, dishes) in an appropriate container for biohazardous materials.

1. Do the first collection $16 \mathrm{~h}$ after the last incubation and add $4 \mathrm{~mL}$ of complete DMEM. Incubate at $37{ }^{\circ} \mathrm{C}, 5 \% \mathrm{CO}_{2}$.

2. Do the second collection $8 \mathrm{~h}$ after the first to the same tube, add $4 \mathrm{~mL}$ of complete DMEM and incubate at $37^{\circ} \mathrm{C}, 5 \% \mathrm{CO}_{2}$.

3. Do the third collection $16 \mathrm{~h}$ after the second to the same tube and discard the dishes. NOTE: Store lentiviral supernatants at $4{ }^{\circ} \mathrm{C}$ after each collection.

12. Filter each lentiviral supernatant using a $0.45 \mu \mathrm{m}$ low-protein binding filter with a cellulose acetate membrane (Table of Materials) to a clean tube.

13. Add a maximum of $15 \mathrm{~mL}$ of filtered supernatant to a centrifugal filter unit with a regenerated cellulose membrane (Table of Materials) and spin at $4,000 \times g$ for $25 \mathrm{~min}$, at $4{ }^{\circ} \mathrm{C}$. Discard flow-through. A viscous liquid containing lentiviruses will remain in the filter unit.

14. Repeat step 3.13 by adding $15 \mathrm{~mL}$ of supernatant on top of the filter unit, until there is no more lentiviral supernatant left. NOTE: When there are only a few milliliters of supernatant to concentrate, decrease the spinning time to 10 min. If there is still extra liquid (non-viscous) on the filter, centrifuge for an additional $10 \mathrm{~min}$.

15. Make aliquots (50-200 $\mu \mathrm{L}$ depending on the initial supernatant volume) of each type of concentrated lentiviruses and store at $-80{ }^{\circ} \mathrm{C}$ for longterm storage (1-2 years) or at $4{ }^{\circ} \mathrm{C}$ for short-term storage (1-2 weeks).

NOTE: Concentrated or non-concentrated lentiviruses can also be used fresh. Do not re-freeze and thaw as this results in reduced titer.

\section{Hemogenic Reprogramming}

NOTE: Use HDFs with a passage number of three (P3) or higher (until P10) to perform reprogramming experiments.

1. Coat a $100 \mathrm{~mm}$ tissue culture-treated dish with $5 \mathrm{~mL}$ of $0.1 \%$ gelatin and incubate at $37^{\circ} \mathrm{C}$ for 20 min. Aspirate the remaining gelatin solution

2. Thaw a fibroblast vial and plate cells in the $0.1 \%$ gelatin-coated dish. Incubate overnight at $37{ }^{\circ} \mathrm{C}, 5 \% \mathrm{CO}_{2}$. If necessary, expand fibroblasts for a longer period of time until the desired passage and confluency are reached.

3. Coat a 6-well tissue culture-treated plate with $500 \mu \mathrm{L}$ of $0.1 \%$ gelatin solution and incubate at $37{ }^{\circ} \mathrm{C}$ for 20 min. Remove extra gelatin

4. Plate HDFs at a density of 150,000 cells per plate $\left(25,000\right.$ cells per well) in $2 \mathrm{~mL}$ of complete DMEM per well. Incubate overnight at $37^{\circ} \mathrm{C}$, $5 \% \mathrm{CO}_{2}$, to allow cell attachment. 
5. Replace medium with $2 \mathrm{~mL}$ of complete DMEM plus $8 \mu \mathrm{g} / \mathrm{mL}$ polybrene. Prepare a 1:1 ratio mix of pool-produced TF lentiviruses and M2rtTA in a new microcentrifuge tube.

NOTE: In this protocol, pool-production of lentiviruses for the three TFs is performed, which, in authors' hands, results in higher reprogramming efficiency. Alternatively, it is suggested to perform a titration of the individual lentiviral particles by $\mathrm{qPCR}{ }^{18}$, on a standard cell line. This will be used to define the volume of individual viruses necessary to meet a multiplicity of infection (MOI) optimal for co-transduction and hemogenic reprogramming.

6. Distribute 10 to $100 \mu \mathrm{L}$ of lentiviral mixture per well, to transduce HDFs. This is day -2 of reprogramming.

NOTE: Defining the optimal volume of lentiviral mix for efficient reprogramming, without compromising cell viability, requires optimization (see Supplementary Figure 1 for more details). HDFs with more than 7 passages may require higher volumes of viruses than cells with lower passages.

7. After $16 \mathrm{~h}$ of incubation, remove viruses and add complete DMEM. Allow cells to recover for $6-8 \mathrm{~h}$.

8. After recovery, aspirate medium and add $2 \mathrm{~mL}$ of complete DMEM with $8 \mu \mathrm{g} / \mathrm{mL}$ polybrene.

9. Do a second transduction as described in step 4.6 and incubate at $37^{\circ} \mathrm{C}, 5 \% \mathrm{CO}_{2}$ for $16 \mathrm{~h}$. This is day -1 of reprogramming. The lentiviral mix can be prepared on day -2 for both transductions and kept at $4{ }^{\circ} \mathrm{C}$.

10. On the next day, remove the viruses and add complete DMEM supplemented with $1 \mu \mathrm{g} / \mathrm{mL}$ DOX. This is day 0 of reprogramming. Incubate at $37^{\circ} \mathrm{C}, 5 \% \mathrm{CO}_{2}$ for $48 \mathrm{~h}$

11. At day 2 of reprogramming, split each well at 1:2 ratio.

1. Aspirate medium and wash cells with $1 \mathrm{~mL}$ of PBS.

2. Aspirate PBS and dissociate cells with $500 \mu \mathrm{L}$ of dissociation solution. Incubate $5-10$ min at $37{ }^{\circ} \mathrm{C}, 5 \% \mathrm{CO}_{2}$.

3. Inactivate the dissociation solution with $1 \mathrm{~mL}$ of complete DMEM and collect cells into a conical tube. Centrifuge at $350 \times g$ for $5 \mathrm{~min}$.

4. Resuspend the pellet in hematopoietic medium (see step 1.3), supplemented with $1 \mu \mathrm{g} / \mathrm{mL}$ DOX, and plate cells into new tissue culture-treated 6 -well plates coated with $0.1 \%$ gelatin to a final volume of $2 \mathrm{~mL}$ per well.

12. Change medium (hematopoietic medium plus DOX) twice a week for the duration of the reprogramming cultures ( 25 days).

13. Analyze resulting reprogrammed cells at different time points by brightfield or fluorescence microscopy (see Supplementary Figure 2), flow cytometry, bulk and single-cell RNA sequencing, and transplantation assays for the acquisition of hematopoietic morphology, presence of endothelial and hematopoietic markers, acquisition of endothelial/hematopoietic gene expression profile and regeneration capacity ${ }^{14}$.

\section{Optimization of Fibroblast Expansion for ChIP-seq Analysis at the Onset of Hemogenic Reprogramming}

1. Plate $300,000 \mathrm{HDFs}(<\mathrm{P} 8)$ in $0.1 \%$ gelatin coated tissue culture-treated 6-well plates with complete DMEM to a final volume of $2 \mathrm{~mL}$ per well. Incubate overnight at $37^{\circ} \mathrm{C}, 5 \% \mathrm{CO}_{2}$.

2. On the following day, replace medium with complete DMEM supplemented with $8 \mu \mathrm{g} / \mathrm{mL}$ polybrene.

3. Transduce cells with individual factors: pFUW-tetO-FOS ${ }^{14}$, pLV-tetO-HA-GFI1B (Addgene \#125599) ${ }^{14}$ and pFUW-tetO-3xFLAG-GATA2 (Addgene $\# 125600)^{14}$ or with a pool of the three factors, plus FUW-M2rtTA at 1:1 ratio. Use 10-20 $\mu \mathrm{L}$ total virus (individual TF + M2rtTA or three TFs + M2rtTA). Incubate cells overnight at $37^{\circ} \mathrm{C}, 5 \% \mathrm{CO}_{2}$.

NOTE: It is recommended to use twelve 6-well plates per condition (for each individual TF and the three TFs combined).

4. Remove lentiviruses and add complete DMEM $16 \mathrm{~h}$ after the first transduction. Let cells recover for $6-8 \mathrm{~h}$.

5. Transduce cells a second time with the same amount of virus per condition and incubate at $37{ }^{\circ} \mathrm{C}, 5 \% \mathrm{CO}_{2}$.

6. On the next day remove viruses and add complete DMEM. Incubate at $37{ }^{\circ} \mathrm{C}, 5 \% \mathrm{CO}_{2}$ for $24 \mathrm{~h}$.

7. Re-plate each well into a $0.1 \%$ gelatin coated tissue culture-treated $100 \mathrm{~mm}$ dish with complete DMEM to a final volume of $10 \mathrm{~mL}$ per dish. This represents approximately a 1:6 passage.

8. Allow cells to grow for 6 days at $37{ }^{\circ} \mathrm{C}, 5 \% \mathrm{CO}_{2}$.

9. On day 6 after re-plating, aspirate medium and add complete DMEM with $1 \mu \mathrm{g} / \mathrm{mL} \mathrm{DOX}$. Incubate cells at $37{ }^{\circ} \mathrm{C}, 5 \% \mathrm{CO}_{2}$ for 2 days.

10. Collect fibroblasts and analyze genomic binding sites of the three TFs transduced individually or in combination, by ChIP-seq 2 days after DOX supplementation ${ }^{14}$.

NOTE: The final seventy-two $100 \mathrm{~mm}$ dishes will contain between $20-50 \times 10^{6}$ cells, sufficient to perform ChIP-seq experiments and replicates.

\section{Representative Results}

A schematic representation of the reprogramming approach using HDFs is illustrated in Figure 1A. Fibroblasts are acquired from commercial sources or collected from human donors and expanded in vitro previous to reprogramming. After plating, cells are transduced twice with GATA2, GFI1B and FOS (and M2rtTA) lentiviruses, and doxycycline is added at day 0 of reprogramming. On day 2, cells are split and plated in hematopoietic medium until day 25 of culture. Reprogrammed cells may be generated at different time points for multiple applications including transplantation in immunocompromised mice, single-cell RNA-sequencing (scRNA-seq) of purified cell populations (day 2 unsorted, day 15

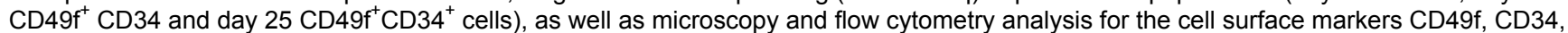
CD9 and CD143. Representative cytometry plots show $\sim 17 \%$ of reprogrammed cells expressing both CD49f and CD9 (Figure 1B, left panel), after 25 days of reprogramming. The majority of double positive cells express CD143 ( $86 \%)$, and a small population express CD34 (0.9\%), suggesting a dynamic hemogenic fate induction. These markers are not activated in M2rtTA transduced HDFs cultured for 25 days (Figure 1B, right panel). Immunofluorescence images confirm expression of CD9 and CD143 in adherent and round cells, morphologically distinct from fibroblasts that are negative for these markers (Figure 1C). Human hemogenic colonies also express CD49f and CD34 ${ }^{14}$. ScRNA-seq analysis of HDFs, day 2 unsorted cells, and purified reprogrammed cells at day $15\left(\mathrm{CD} 49 \mathrm{f}^{+} \mathrm{CD} 34^{-}\right)$and day $25\left(\mathrm{CD} 49 \mathrm{f}^{+} \mathrm{CD} 34^{+}\right)$show a stepwise increase in CD49f, CD9 and CD143 expression from day 2 to day 25. CD49f and CD9 positive cells appear first during the reprogramming process, between day 2 and 15, indicating that these molecules may represent markers of early human hemogenesis. CD143 expression starts to be 
detected at day 15 and CD34 expressing cells are detected only at later time points (day 25). CD34 ${ }^{+}$umbilical cord blood (UCB) cells were used as reference (Figure 1D).

Figure 2A describes a modified protocol to generate sufficient number of cells for ChIP-seq analysis at the initial stages of hemogenic reprogramming (day 2). First, HDFs are plated at a density two times higher than in the standard protocol $(300,000$ cells versus 150,000 cells per plate). After transduction, each well is re-plated into a $100 \mathrm{~mm}$ dish allowing cells to expand for 6 days before supplementing medium with DOX. Cells are analyzed 2 days after adding DOX and consequent TF expression. Figure 2B shows genome browser profiles of GATA2 binding to genomic regulatory regions of ITGA6 and ACE when cells are co-transduced with the three factors (3TFs) or GATA2 individually. GATA2 also binds to open chromatin regions of $C D 9$ and $C D 34$ genes $^{14}$.
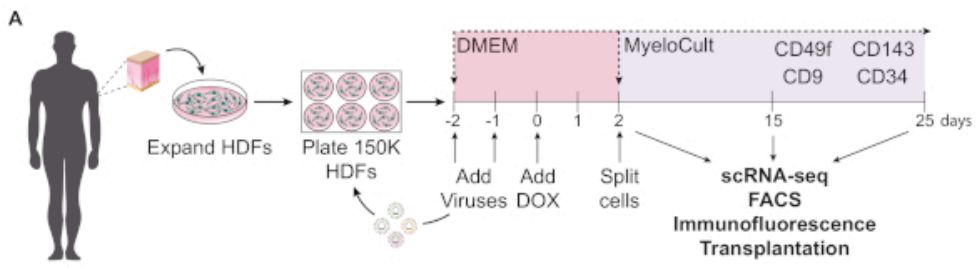

B
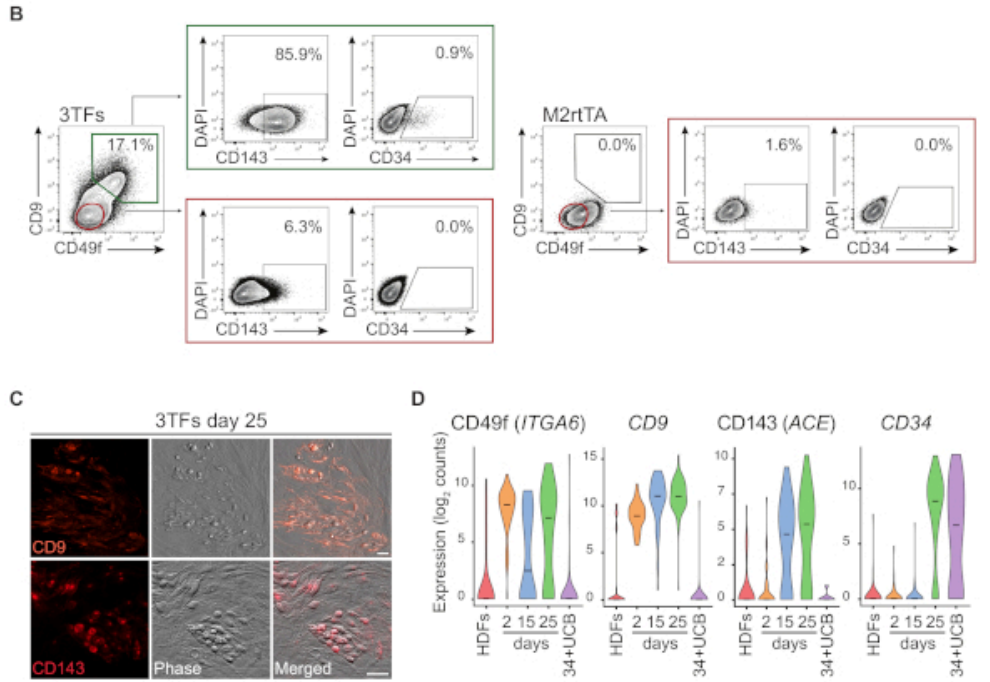

Figure 1: Induction of hemogenic fate in human dermal fibroblasts. (A) Experimental strategy for hemogenic reprogramming of human dermal fibroblasts (HDFs). Fibroblasts from skin punch biopsies are collected from donors, expanded and transduced with GATA2, GFI1B, FOS and M2rtTA lentiviruses. Doxycycline (DOX) is added to the culture at day 0 of reprogramming and cells are analyzed at several time points until day 25. scRNA-seq, single cell RNA-sequencing. FACS, Fluorescence-Activated Cell Sorting. (B) Gating strategy used to evaluate the expression of hemogenic/hematopoietic markers by flow cytometry at day 25 after transduction with the three transcription factors (3TFs). Cytometry plots show percentage of double positive cells for CD49f and CD9, gated in the live-cell population (DAPI-negative). Within the double positive population, the expression of CD143 and CD34 is shown. HDFs transduced only with M2rtTA virus under the same culture conditions are used as control. (C) Immunofluorescence images of day 25 reprogrammed colonies confirming the expression of CD9 (upper panel) and CD143 (lower panel). Cells were stained with antibodies (Table of Materials) diluted 1:100 in PBS/2\% FBS with mouse serum, incubated 20 min at $37{ }^{\circ} \mathrm{C}, 5 \% \mathrm{CO}_{2}$, washed three times and imaged in PBS/2\% FBS. Phase, phase-gradient contrast. Scale bars $=50 \mu \mathrm{m}$. (D) ScRNA-seq analysis of 253 cells at different time points. Expression of ITGA6, CD9, ACE and CD34 is activated during reprogramming. Cells are collected at day 2 (unsorted), day 15 (CD49f ${ }^{+}$CD34 ) and day 25 (CD49f ${ }^{+}$CD $\left.34^{+}\right)$. HDFs and CD34 ${ }^{+}$umbilical cord blood (34+UCB) cells are used as references. Please click here to view a larger version of this figure. 
A

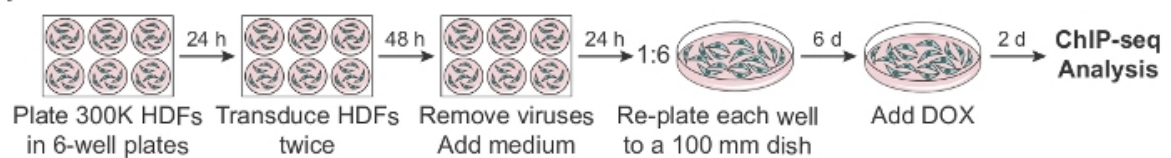
in 6-well plates twice Add medium to a $100 \mathrm{~mm}$ dish

B
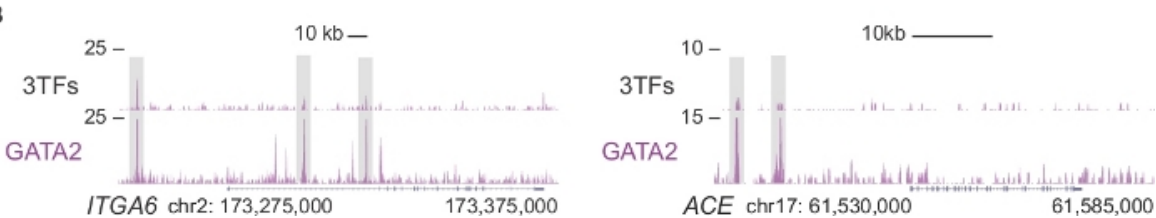

Figure 2: Expansion of human dermal fibroblasts for ChIP-seq analysis. (A) Experimental strategy depicting a modified protocol to generate high numbers of transduced human dermal fibroblasts (HDFs) for ChIP-seq at day 2 of reprogramming. 300,000 cells are plated in 6-well plates and transduced twice with individual factors (pFUW-tetO-FOS, pLV-tetO-HA-GFI1B or pFUW-tetO-3xFLAG-GATA2) or a combination of the three factors (plus M2rtTA). After removing viruses, fibroblasts are expanded for six days in $100 \mathrm{~mm}$ dishes. Doxycycline (DOX) is added at day 0 and cells are collected two days after DOX addition. (B) Genome browser profiles highlighting GATA2-binding sites (grey boxes) at ITGA6 and ACE loci two days after transduction with the three transcription factors (3TFs) or with GATA2 alone. The total number of mapped reads is represented on the y-axis. Please click here to view a larger version of this figure.

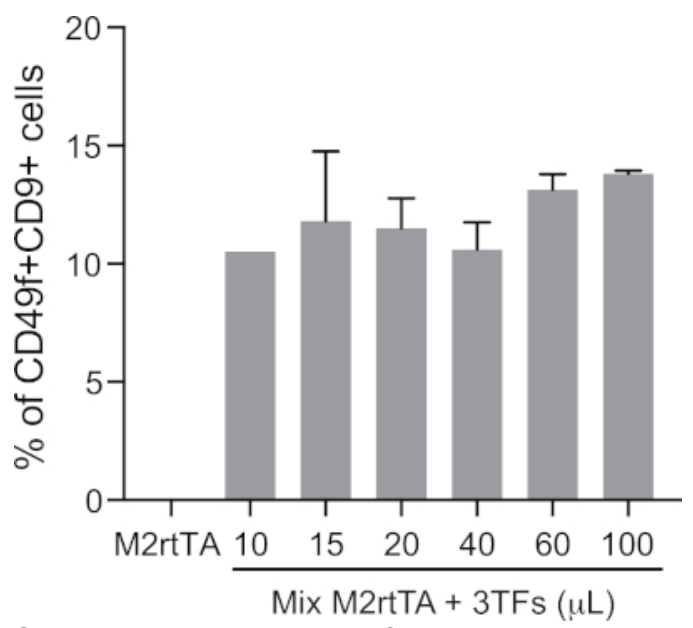

Supplementary Figure 1: Defining an optimized lentiviral volume for efficient hemogenic reprogramming. Increasing volumes of concentrated (10 to $100 \mu \mathrm{L}$ ) pool-produced lentiviral particles (3TFs: GATA2, GFI1B and FOS) are used to transduce human dermal fibroblasts (HDFs), together with M2rtTA at a ratio of 1:1, following steps 4.5-4.12 of the protocol. Reprogrammed cells are analyzed at day 25 to define an optimal volume of transduction for hemogenic reprogramming, given by the percentage of $\mathrm{CD} 49 \mathrm{f}^{+} \mathrm{CD} 9^{+}$cells gated in live-cells (DAPI-negative). Cell viability can be assessed by quantifying the absolute number of live cells at day 25 . HDFs transduced with M2rtTA (100 $\mu \mathrm{L})$ are used as negative control. Please click here to view a larger version of this figure.

Day -2

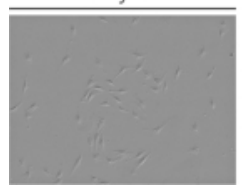

\section{Supplen}

Day 2

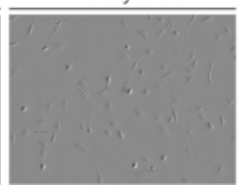

Day 15

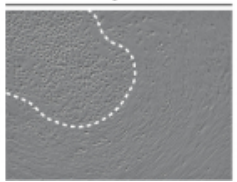

Day 25

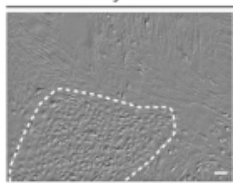
Fibroblast (HDF) cultures are imaged at the day of the first transduction (day -2), when DOX is added to the cultures (day 0), two days (day 2) and fifteen days (day 15) after DOX supplementation, and at the end-point of the experiment (day 25). Hemogenic colonies at days 15 and 25 are highlighted. Scale bars $=100 \mu \mathrm{m}$. Please click here to view a larger version of this figure.

\section{Discussion}

In this article, a method is described to generate hematopoietic progenitor cells directly from human fibroblasts, which go through an HP cell intermediate, similarly to definitive $\mathrm{HSCs}^{14}$.

Pool-production of lentiviral particles encoding GATA2, GFI1B and FOS was preferred over individual production, since in our hands it results in higher reprogramming efficiencies (unpublished data). Lentiviruses, as members of the Retroviridae family, normally contain two copies of positive single-stranded RNA ${ }^{19}$. The increased reprogramming efficiency may be due to packaging of two different transgenes in the same lentiviral particle, resulting in increased number of cells co-transduced with the three transcription factors. To ensure the success of this protocol, it is necessary to transduce HDFs with adequate amount of virus depending on the cell passage to obtain an optimal balance between reprogramming efficiency and cell viability, as recommended in step 4.6. Moreover, fresh non-concentrated viruses can be used. It is recommended to transduce cells with $0.5-3 \mathrm{~mL}$ of 3 TFs pool and M2rtTA. Also, cell density should be adjusted according to the application. 
150000 HDFs per 6-well plate (step 4.4) provided the optimal density to perform FACS, transplantation and flow cytometry analysis of reprogrammed cells. For ChIP-seq experiments, more cells were required from the beginning (step 5.1). It is important to check cells regularly for morphological changes and replace hematopoietic medium twice a week to support the emergence of induced hematopoietic cells. Addition of hematopoietic cytokines or co-culture in feeder layers may increase reprogramming efficiency.

With this method, we can identify new hematopoietic markers that are dynamically expressed during hemogenic reprogramming. CD9, which was shown to be up-regulated in reprogrammed cells at the transcriptional level ${ }^{14}$, is rapidly expressed at the cell surface in the initial phases of reprogramming together with CD49f and CD143, serving as a novel marker of human HSC precursors. We also show that ITGA6 and ACE are direct targets of GATA2 during the initial stages of hemogenic reprogramming, in addition to $C D 9$ and $C D 34^{14}$, providing a direct mechanistic link between human hemogenic precursor phenotype and GATA2.

One advantage of this system resides in the use of relatively homogeneous fibroblast cultures. While PSCs are easily expanded and maintained in vitro, differentiation protocols generate heterogeneous populations that include hematopoietic progenitors, which engraft poorly, 5,7 . Moreover, there is a risk of tumorigenesis when transplanting PSC-derived HSPCs, since undifferentiated PSCs may still remain in culture even after employing differentiation protocols. Alternatively to fibroblasts, direct reprogramming to HSCs has been applied to blood-committed progenitors ${ }^{20}$ and endothelial cells ${ }^{21}$. However, starting with blood-restricted progenitor cells hinders therapeutic application of the resulting HSCs if the patient carries mutations that affect the stem/progenitor hematopoietic population ${ }^{22}$. In the case of endothelial cells, these are more difficult to obtain compared to fibroblasts, and constitute a very heterogeneous cell population in terms of phenotype, function and structure, which are organdependent $^{23}$. Other studies have succeeded in reprogramming mouse fibroblasts into engraftable hematopoietic progenitors ${ }^{24,25}$ yet, so far, no other protocol describes the generation of HSPC-like cells from human fibroblasts.

This approach, coupled with pharmacological inhibition, gene knock-out, or knock-down permits to define individual or combination of factors that are required to directly induce human HSCs. Employing high efficiency screening methodologies based on recent CRISPR-Cas9 technologies in HDFs prior to reprogramming, represents an exciting endeavor for defining novel regulators of human definitive hematopoiesis. In the future, reprogramming non-blood related human cell types such as fibroblasts will serve as a platform to generate healthy patient-tailored hematopoietic progenitor cells for clinical applications.

\section{Disclosures}

The authors have nothing to disclose.

\section{Acknowledgments}

The Knut and Alice Wallenberg foundation, the Medical Faculty at Lund University and Region Skåne are acknowledged for generous financial support. This work was supported by a grant from Olle Engkvists Stiftelse (194-0694 to Filipe Pereira) and PhD scholarships from Fundação para a Ciência e Tecnologia (PTDC/BIM-MED/0075/2014 to Filipe Pereira, and SFRH/BD/135725/2018 and SFRH/BD/51968/2012 to Rita Alves and Andreia Gomes). This study was also supported by funds from NIH and NYSTEM (1R01HL119404 and C32597GG to Kateri A. Moore).

\section{References}

1. Ivanovs, A. et al. Highly potent human hematopoietic stem cells first emerge in the intraembryonic aorta-gonad-mesonephros region. Journal of Experimental Medicine. 208, 2417-2427 (2011).

2. Tavian, M., Biasch, K., Sinka, L., Vallet, J., Péault, B. Embryonic origin of human hematopoiesis. International Journal of Developmental Biology. 1065, 1061-1065 (2010).

3. Medvinsky, A., Rybtsov, S., Taoudi, S. Embryonic origin of the adult hematopoietic system: advances and questions. Development. 138, 1017-1031 (2011)

4. Ivanovs, A. et al. Human haematopoietic stem cell development: from the embryo to the dish. Development. 144, 2323-2337 (2017).

5. Daniel, M. G., Pereira, C.-F., Lemischka, I. R., Moore, K. A. Making a Hematopoietic Stem Cell. Trends in Cell Biology. 26, 202-214 (2016).

6. Vo, L., Daley, G. De novo generation of HSCs from somatic and pluripotent stem cell sources. Blood. 125, 2641-2648 (2015).

7. Rafii, S. et al. Human ESC-derived hemogenic endothelial cells undergo distinct waves of endothelial to hematopoietic transition. Blood. 121, 770-781 (2013).

8. Ebina, W., Rossi, D. J. Transcription factor-mediated reprogramming toward hematopoietic stem cells. EMBO Journal. 34, 694-709 (2015).

9. Sugimura, R. et al. Haematopoietic stem and progenitor cells from human pluripotent stem cells. Nature. 545, 432-438 (2017).

10. Pereira, C. F. et al. Induction of a Hemogenic Program in Mouse Fibroblasts. Cell Stem Cell. 13, 205-218 (2013).

11. Pereira, C. F. et al. Hematopoietic Reprogramming In vitro Informs In Vivo Identification of Hemogenic Precursors to Definitive Hematopoietic Stem Cells. Developmental Cell. 36, 525-539 (2016).

12. Notta, F. et al. Isolation of Single Human Hematopoietic Stem Cells Capable of Long-Term Multilineage Engraftment. Science. 333, 218-221 (2011).

13. Sinka, L., Biasch, K., Khazaal, I., Péault, B., Tavian, M. Angiotensin-converting enzyme (CD143) specifies emerging lympho-hematopoietic progenitors in the human embryo. Blood. 119, 3712-3724 (2012).

14. Gomes, A. M. et al. Cooperative Transcription Factor Induction Mediates Hemogenic Reprogramming. Cell Reports. 25, 2821-2835 (2018).

15. Karlsson, G. et al. Report The Tetraspanin CD9 Affords High-Purity Capture of All Murine Hematopoietic Stem Cells. Cell Reports. 4, 642-8 (2013).

16. Leung, K. T. et al. The tetraspanin CD9 regulates migration, adhesion, and homing of human cord blood CD34+ hematopoietic stem and progenitor cells. Blood. 117, 1840-1851 (2011).

17. Hockemeyer, D. et al. A drug-inducible system for direct reprogramming of human somatic cells to pluripotency. Cell Stem Cell. 3, 346-353 (2008). 
18. Kutner, R. H., Zhang, X., Reiser, J. Production, concentration and titration of pseudotyped HIV-1-based lentiviral vectors. Nature Protocols. 4, 495-505 (2009).

19. Suzuki, Y. S., Suzuki, Y. Gene Regulatable Lentiviral Vector System. In Viral Gene Therapy. Edited by Ke, X., 286-308, IntechOpen. (2011).

20. Riddell, J. et al. Reprogramming committed murine blood cells to induced hematopoietic stem cells with defined factors. Cell. 157, 549-564 (2014).

21. Lis, R. et al. Conversion of adult endothelium to immunocompetent haematopoietic stem cells. Nature. 545, 439-445 (2017).

22. Pereira, C., Lemischka, I. R., Moore, K. 'From blood to blood': de-differentiation of hematopoietic progenitors to stem cells. EMBO Journal. 33, 1511-1513 (2014).

23. Nolan, D. J. et al. Molecular Signatures of Tissue-Specific Microvascular Endothelial Cell Heterogeneity in Organ Maintenance and Regeneration. Developmental Cell. 26, 204-219 (2013).

24. Batta, K., Florkowska, M., Kouskoff, V., Lacaud, G. Direct Reprogramming of Murine Fibroblasts to Hematopoietic Progenitor Cells. Cell Reports. 9, 1871-1884 (2014).

25. Cheng, H. et al. Reprogramming mouse fibroblasts into engraftable myeloerythroid and lymphoid progenitors. Nature Communications. 7, 115 (2016). 